\title{
Hidradenocarcinoma in a young Caucasian male, arising from a metatarsophalangeal joint and presenting with inguinal lymphadenopathy
}

\author{
Chii Yang Kuah" ${ }^{*}$, Jaslyn Ju Lia Gan ${ }^{2}$ and Steve Nicholson ${ }^{1}$ \\ ${ }^{1}$ Department of Oncology, Southend Hospital, Prittlewell Chase, Southend-on-Sea, UK \\ ${ }^{2}$ Lister Hospital, Coreys Mill Ln, Stevenage SG1 4AB, UK
}

\begin{abstract}
We report a case of a 25-year-old male with primary hidradenocarcinoma arising from the medial aspect of the left metatarsophalangeal joint who presented with palpable inguinal lymphadenopathy. The patient was able to give a corroborated history of the primary lesion being present for 4 years prior to the development of his groin swelling. Initial biopsy of the primary lesion suggested a malignant adnexal tumour. He underwent wide local excision of the primary lesion and therapeutic inguinal lymph node dissection. Review of the excision specimens established the diagnosis of hidradenocarcinoma. We discuss this case due to the rarity of the site of the tumour and demographics of the patient. There is limited data available on hidradenocarcinoma and there is no consensus on what should constitute standard identification and management.
\end{abstract}

\section{Introduction}

Hidradenocarcinoma, the malignant form of hidradenoma, is a very rare sweat gland cancer that is estimated to represent less than $0.001 \%$ of all cancers, and $0.01 \%$ of skin cancers [1]. Hidradenocarcinomas are most often reported presenting on the head and neck [1,2]. Very few reports describe hidradenocarcinoma developing on the extremities [2]. We describe a presentation that is unusual in its primary site and the demographics of the patient.

The literature provides limited data on hidradenocarcinoma and there is no consensus on standard identification and management. Approximately eighty cases of hidradenocarcinoma have been described in the English literature, the majority focusing on histology, with limited clinical detail $[2,3]$.

\section{Case report}

A 25-year-old male presented to his general practitioner with a palpable left inguinal mass measuring $1 \times 2 \mathrm{~cm}$. Ultrasound scan confirmed this to be inguinal lymphadenopathy. Clinical examination revealed a $3.5 \times 1.5 \times 2.5 \mathrm{~cm}$, cystic, smoothly-elevated mass in the plantar aspect of the left first metatarsophalangeal joint. The patient described the foot mass as having been present for four years, corroborated by a former girlfriend, and the inguinal mass for approximately one year. CT scan of chest, abdomen and pelvis showed the enlarged left inguinal lymph node with no other abnormalities (Figure 1), and MRI of the foot (Figure 2) showed a lesion in-keeping with a diagnosis of sarcoma. Biopsy, however, was consistent with an adnexal cancer, without characteristics to permit further refinement of diagnosis. Treatment comprised excision of the primary with reconstruction, together with therapeutic left inguinal lymph node dissection yielding one involved node. Histological examination of the full resection specimen enabled a definitive diagnosis of hidradenocarcinoma. The patient has been on clinical surveillance for one year, with no evidence of recurrence.

\section{Discussion}

Hidradenocarcinoma usually presents on sun-exposed areas (notably the head and neck region) and is rarely reported on the distal extremities [2]. It is difficult to distinguish the tumour by appearance alone. It mimics a benign solitary skin lesion, starting as a solitary firm nodule or an erythematous plaque with normal overlying skin. The lesion may expand over a variable period ranging from months to decades. Most recorded cases to date have demonstrated progression, but most patients are asymptomatic, with a minority experiencing discomfort, ulceration, pain or bleeding of the skin lesion $[1,2]$. The asymptomatic nature of the tumour and benign appearances may contribute to it underdiagnoses.

This tumour usually presents in the third to sixth decade, but it has been reported in children [3]. There is a suggestion that it is more common in females [2].

Diagnosis and clinical decision-making are based on histology (including immunohistochemical analysis) and imaging to define the extent of metastatic spread [3]. Sentinel lymph node biopsy may be useful in the identification of subclinical spread to surrounding lymph nodes [1]. Bogner et al discuss two patients who had sweat gland cancer, neither of whom demonstrated clinical signs of metastasis. Both underwent wide local excisions with concurrent sentinel lymph node biopsy and both showed micrometastatic spread to the draining lymph

${ }^{\star}$ Correspondence to: Chii Yang Kuah, 217, Whitehouse Apartments, 9 Belvedere Road SE1 8YR, London, UK, E-mail: james.kcy@hotmail.com

Key words: hidradenocarcinoma, wide local excision, metastatic rate, lymph nodes, sentinel node biopsy

Received: August 17, 2018; Accepted: October 04, 2018; Published: October 29,2018 


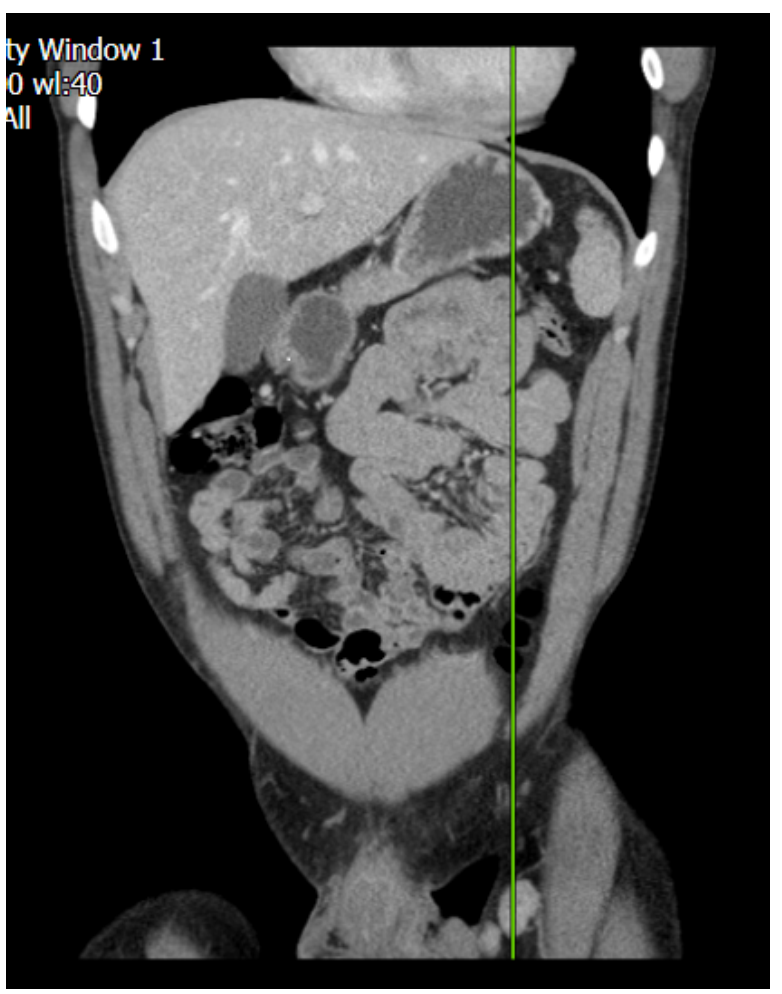

Figure 1. CTCAP image showing an enlarged left inguinal lymph node with short axis reaching up $2 \mathrm{~cm}$

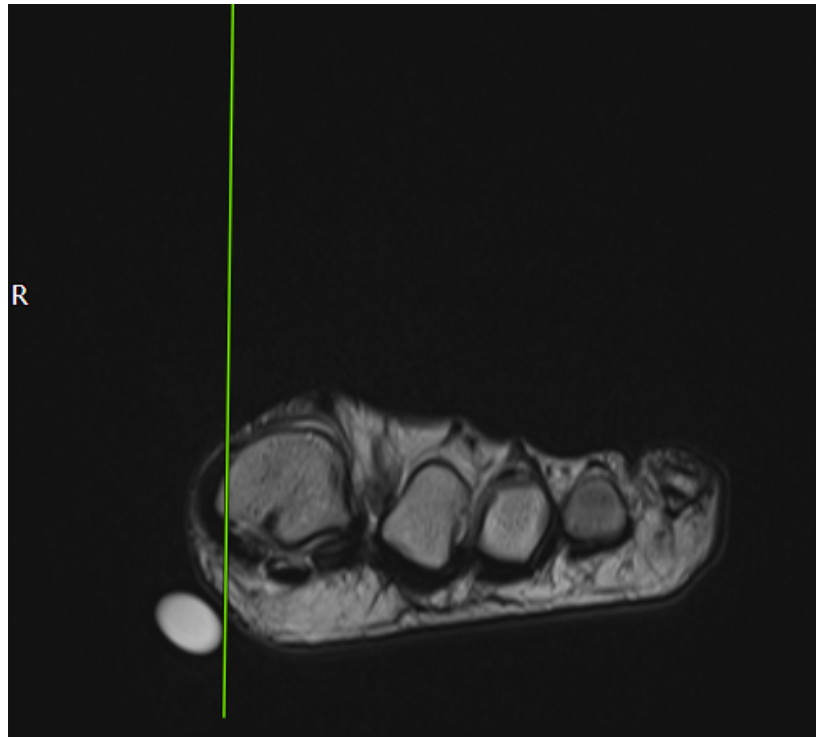

Figure 2. Foot MRI image showing $3.5 \times 1.5 \times 2.5 \mathrm{~cm}$, cystic, smoothly-elevated mass in the plantar aspect

node basin. They suggest that sentinel lymph node biopsy might have value as a staging tool [4].

Evidence guiding the management of hidradenocarcinoma is limited: wide local excision with or without prophylactic lymphadenectomy is the treatment most often described for clinically node-negative disease [1]. The perception that this is a rapidly-growing cancer has led to the recommendation that wide local excision should be performed as a matter of urgency [2,3]. The role of prophylactic lymph node dissection is controversial, with no evidence to suggest that it increases the disease-free interval. Some authors recommend prophylactic lymphadenectomy on the basis of the aggressive and high metastatic potential of the tumour [5].

The primary hidradenocarcinoma described here behaved indolently and had been present for at least 3 years before metastasising to the inguinal lymph node basin. Invasion of locoregional lymph nodes is a standard indication for therapeutic lymph node dissection, and our patient was therefore managed by wide local excision with therapeutic lymphadenectomy [6].

Hidradenocarcinoma was initially thought to be resistant to radiotherapy, but there is some suggestion that post-operative radiotherapy may improve local control: Harari et al. reported a series of three patients where the primary lesion and the draining lymph node basin were treated with external beam radiotherapy [7]. The patientsmaintained remission for up to 35 months.

The role of chemotherapy in the adjuvant setting is uncertain, but systemic therapy is usually considered in patients with metastatic disease. Fluoropyrimidine-based regimens are most often reported, with capecitabine or intravenous 5-fluorouracil (5FU) popular as firstline treatment $[8,9]$. A full survey of systemic therapy is beyond the scope of this report, but successful systemic therapy with non-cytotoxic agents has been reported, including the vascular endothelial growth factor (VEGF) inhibitor sunitinib [10].

Early diagnosis is generally considered critical to successful treatment outcome and preservation of quality of life, but five-year postsurgical survival rate is less than $30 \%$, with local recurrence rates ranging from 10 to $50 \%[2,3,8]$. Our patient is still in remission three years post-surgery with no adjuvant therapy.

\section{Conclusion}

Hidradenocarcinoma is difficult to identify through appearance and is often confused with benign lesions. There are very few reported cases and no prospective studies to inform epidemiology and management options. Clinical trials in rare cancers are particularly challenging, and a novel approach to trial design would be essential in a tumour with the rarity of hidradenocarcinoma.

\section{References}

1. Soni A, Nupur B, Vivek K, Ashok K (2015) Current management approach to hidradenocarcinoma: A comprehensive review of the literature. Ecancermedicalscience 9: 517. [Crossref]

2. Gauerke S, Driscoll J (2010) Hidradenocarcinomas: A brief review and future directions. Arch Pathol Lab Med 134: 781-85. [Crossref]

3. Genetic and Rare Diseases Information Center (GARD) - An NCATS program Hidradenocarcinoma.

4. Bogner N, Douglas F, Lori L, Paulino A, Biermann JS, et al. (2003) Lymphatic mapping and sentinel lymph node biopsy in the detection of early metastasis from sweat gland carcinoma. Cancer 97: 2285-2289. [Crossref]

5. Shu K, Xiao Q, Buchele F, Zhang S, Jiang W, et al. (2012) Diagnosis and treatment of clear cell hidradenocarcinoma of the scalp. J Huazhong Univ Sci Technolog Med Sci 32: 931-936. [Crossref]

6. Sinhasan S, Harthimath B, Sylvia M, Ramachandra V (2015) Metastatic malignant nodular hidradenoma. A rare case report with review of literature. Clin Cancer Investig J 4: 651-654.

7. Harari PM, Shimm DS, Bangert JL, Cassady JR (1990) The role of radiotherapy in the treatment of malignant sweat gland neoplasms. Cancer 65: 1737-1740. [Crossref]

8. Amel T, Gharbi O, Hammedi F, Hochlef M, Ben A, et al. (2009) Metastatic hidradenocarcinoma: surgery and chemotherapy. N Am J Med Sci 1: 372-374. [Crossref] 
Kuah CY (2018) Hidradenocarcinoma in a young Caucasian male, arising from a metatarsophalangeal joint and presenting with inguinal lymphadenopathy

9. Lerner A, Beckford A, Ugent S, Goldberg L, Jalisi S, et al. (2011) Complete response of metastatic malignant hidradenocarcinoma to capecitabine treatment. Arch Dermatol 147: 998-999. [Crossref]
10. Battistella M, Mateus C, Lassau N, Chami L, Boukoucha M, et al. (2010) Sunitinib efficacy in the treatment of metastatic skin adnexal carcinomas: report of two patients with hidradenocarcinoma and trichoblastic carcinoma. J Eur Acad Dermatol Venereol 24: 199-203. [Crossref]

Copyright: (C2018 Kuah CY. This is an open-access article distributed under the terms of the Creative Commons Attribution License, which permits unrestricted use, distribution, and reproduction in any medium, provided the original author and source are credited. 